\title{
Cáncer gástrico. De la metaplasia intestinal a la terapéutica endoscópica en enfermedad avanzada
}

\author{
Guido Grajales-Figueroa* \\ Instituto Nacional de Ciencias Médicas y Nutrición Salvador Zubirán, Ciudad de México, México
}

\begin{abstract}
Resumen
Se describen los trabajos más destacados sobre el tema presentados durante la DDW 2019, en San Diego, Estados Unidos.

Palabras clave: Cáncer gástrico. Metaplasia intestinal. Disección endoscópica de la submucosa.
\end{abstract}

En la más reciente Semana de Enfermedades Digestivas (DDW 2019), se presentaron alrededor de 40 trabajos originales sobre cáncer gástrico (CG), lesiones premalignas y terapia endoscópica en enfermedad avanzada. A continuación se describen los trabajos que se consideraron más destacados sobre el tema.

En primer lugar, un grupo de investigadores estadounidenses encabezado por el Dr. P. Sharma presentó una revisión sistemática con metaanálisis que tuvo por objetivo estimar la incidencia de CG en pacientes con metaplasia intestinal gástrica (MIG) ${ }^{1}$. Para ello realizaron una revisión de la bibliografía publicada en inglés de 1996 a 2018, sobre pacientes con MIG, duración de su seguimiento y casos incidentes de CG. Los datos fueron clasificados con base en la incidencia de CG por país en $<7,7-14$ y $>14$ por 100 personas-año. El desenlace primario fue la incidencia anual de CG en pacientes con MIG por 100 personas-año y el secundario fue la incidencia anual acumulada estratificada basada en la incidencia de CG de los diferentes países. Se incluyeron 15 estudios con 81,552 pacientes y 457,089 personas-año de seguimiento. La edad promedio de los pacientes fue de 61.1 años y el promedio de seguimiento fue 5.2 años. La incidencia anual acumulada de CG en pacientes con
MIG fue de 0.31 (IC 95\%: 0.21-0.45, I2 92\%) por 100 personas-año. Al estratificar por incidencia de CG dependiendo del país de origen del estudio, la incidencia anual acumulada para $<7,7-14,>14$ casos de CG por personas-año fue de 0.12 (IC 95\%: 0.09-0.15), 0.31 (IC 95\%: 0.25-0.38) y 0.81 (IC 95\%: 0.58-1.2) por 100 personas año, respectivamente, con un incremento estadísticamente significativo en la tendencia $(p<0.001)$. Los resultados de este metaanálisis son valiosos tomando en cuenta el gran número de pacientes incluidos así como el origen variado de los estudios y reproducen los resultados identificados en estudios individuales europeos, asiáticos y norteamericanos en donde se han identificado tasas de progresión a CG entre 0.1 y 0.2 por 100 personas-año y hasta 0.3 si se incluye displasia de alto grado ${ }^{2-6}$. Así mismo, con este estudio se ratifica el conocimiento previo sobre el riesgo incrementado de CG que tienen los pacientes con MIG, sin embargo, se hace evidente también que el riesgo absoluto es menor en regiones en donde la incidencia basal de cáncer gástrico es baja como Norteamérica y Europa. Es importante señalar que en este metaanálisis no se tomaron en cuenta factores de riesgo para progresión a CG adicionales a la MIG, además de que no se hizo una

\section{Correspondencia:}


estratificación sobre la extensión o tipo de MIG, lo cual impacta en el riesgo de progresión.

Por otro lado, en cuanto al tratamiento del cáncer gástrico temprano (CGT), es conocido que en Asia oriental y algunos países europeos, la disección endoscópica de la submucosa (DES) se considera el de elección cuando se cumplen los criterios tradicionales o extendidos. Lo anterior debido a que se han realizado estudios comparativos entre cirugía y DES con resultados similares, prefiriéndose la DES por menor invasividad y costos. Sin embargo, en Norteamérica la DES no ha sido del todo aceptada y no se han llevado a cabo estudios comparativos entre estas técnicas utilizando criterios estrictos. Por ello, Kerdsirichairat, et al. llevaron a cabo un estudio multicéntrico de 16 hospitales norteamericanos (14 estadounidenses y 2 canadienses), comparando de forma retrospectiva los desenlaces de pacientes con CGT que fueron sometidos a cirugía o DES de diciembre de 2004 a febrero de 2018, con seguimiento hasta octubre de $2018^{7}$. Se excluyeron los pacientes que no cumplieran con los criterios tradicionales o extendidos, con evidencia de metástasis ganglionares o a distancia, sin resección curativa o con seguimiento menor a 6 meses. Los desenlaces primarios fueron sobrevida global, sobrevida específica para cáncer y sobrevida libre de recurrencia. Se analizaron en total 75 pacientes ( 38 tratados con DES y 37 tratados con cirugía). No se encontraron diferencias significativas entre ambas técnicas en cuanto a los desenlaces primarios. No se identificaron de forma subsecuente metástasis ganglionares 0 a distancia en ninguno de los grupos y tampoco se identificaron factores demográficos, relacionados con la técnica o histológicos asociados con los desenlaces primarios. Este estudio demuestra que la DES puede llevarse a cabo en Norteamérica y ser considerada una opción a la cirugía con desenlaces oncológicos similares. Por otro lado, estos resultados motivan a que en nuestro país, en los centros donde se hace DES, se considere a esta técnica dentro del armamentario para el tratamiento de los pacientes con CGT.

Finalmente, un aspecto importante en el manejo de pacientes con cáncer gástrico avanzado es la paliación de la obstrucción del tracto de salida del estómago (OTSE). Orr y colaboradores presentaron un estudio retrospectivo que tuvo por objetivo comparar las tasas de disfunción del stent y de reintervención en pacientes con stent enteral paliativo por OTSE debido a adenocarcinoma pancreático (ACP) versus otras etiologías malignas ${ }^{8}$. Se incluyeron 43 pacientes, de los cuales 27 (62.8\%) tuvieron ACP, mientras que 16 (37.2\%) tuvieron otra etiología maligna causante de la OTSE. Siete pacientes requirieron reintervención por OTSE sintomática. De estos pacientes, $14.3 \%$ (1/7) tuvo ACP y $85.7 \%$ (6/7) tuvo OTSE por otra etiología maligna $(p<0.01)$. Después de la colocación del stent enteral, 96\% (26/27) de los pacientes con ACP no requirieron intervención adicional. En el análisis multivariado, controlando para radioterapia como potencial confusor, los pacientes con ACP tuvieron significativamente menores probabilidades de requerir reintervención en comparación con pacientes con otros tipo de cáncer causantes de OTSE (OR 0.064, IC 95\%: 0.01-0.60). Este trabajo demuestra que la colocación de stent enteral en pacientes con OTSE es una buena opción para la paliación de pacientes con ACP y que en caso de presentar otra etiología como causante de la OTSE se requerirá de más reintervenciones y considerar métodos endoscópicos alternativos.

\section{Conflicto de intereses}

No existen conflictos de interés. Sin patrocinio de la industria.

\section{Bibliografía}

1. Parasa S, Thoguluva Chandrasekar V, Kennedy KF, et al. The incidence of gastric cancer in patients with gastric intestinal metaplasia: A systematic review and meta-analysis. Accepted abstracts DDW 2019. San Diego, CA. DDW 2019.

2. Song H, Ekheden IG, Zheng Z, et al. Incidence of gastric cancer among patients with gastric precancerous lesions: observational cohort study in a low risk Western population. BMJ. 2015;351:h3867. Epub 2015 Jul 27.

3. de Vries AC, van Grieken NC, Looman CW, et al. Gastric cancer risk in patients with premalignant gastric lesions: a nationwide cohort study in the Netherlands. Gastroenterology. 2008;134(4):945.

4. den Hollander WJ, Holster IL, den Hoed CM, et al. Surveillance of premalignant gastric lesions: a multicentre prospective cohort study from low incidence regions. Gut. 2019;68(4):585. Epub 2018 Jun 6.

5. Reddy KM, Chang JI, Shi JM, et al. Risk of Gastric Cancer Among Patients With Intestinal Metaplasia of the Stomach in a US Integrated Health Care System. Clin Gastroenterol Hepatol. 2016;14(10):1420. Epub 2016 Jun 16.

6. Li D, Bautista MC, Jiang SF, et al. Risks and Predictors of Gastric Adenocarcinoma in Patients with Gastric Intestinal Metaplasia and Dysplasia: A Population-Based Study. Am J Gastroenterol. 2016;111(8):1104. Epub 2016 May 17.

7. Kerdsirichairat $\mathrm{T}$, Wang $\mathrm{R}$, Aihara $\mathrm{H}$, et al. Outcomes of endoscopic submucosal dissection versus surgery in early gastric cancer meeting standard and expanded indications: A multicenter North American cohort. Accepted abstracts DDW 2019. San Diego, CA. DDW 2019.

8. Orr J, Lockwood RA, Gamboa A, et al. Enteral stents for malignant gastric outlet obstruction: Low reintervention rates for obstruction due to pancreatic ductal adenocarcinoma versus other malignant etiologies. Accepted abstracts DDW 2019. San Diego, CA. DDW 2019. 